\title{
The First-principles Calculations on the Half-metallic Properties of (001) and (110) Surfaces of Zinc-blende YC
}

\author{
Beata Bialek and Jae Il Lee* \\ Department of Physics, Inha University, Incheon 402-751, Korea
}

(Received 5 December 2014, Received in final form 21 February 2015, Accepted 26 February 2015)

\begin{abstract}
We investigated the half-metallicity and magnetism at the (001) and (110) surfaces of YC in zinc-blende structure by using the all-electron full-potential linearized augmented plane wave method within the generalized gradient approximation. From the calculated local density of states, we found that neither (001) nor (110) surface preserves the half-metallicity. While the magnetic moment of $Y$ atom in the YC bulk is $0.116 \mu_{B}$, it is $0.057 \mu_{\mathrm{B}}$ at the topmost layer of Y-terminated (001) surface. On the contrary, C-terminated (001) YC surface exhibits stronger magnetism than the bulk structure; the calculated magnetic moment on topmost $\mathrm{C}$ atom is $1.084 \mu_{\mathrm{B}}$, while that of $\mathrm{C}$ atom in the bulk structure is $0.423 \mu_{\mathrm{B}}$. The magnetic properties of the non-polar (110) YC surface are slightly enhanced as compared with the bulk structure.
\end{abstract}

Keywords : half-metal, surface magnetism, first-principles method

\section{Introduction}

Half-metals are substances that act as conductors to electrons with one spin orientation but as insulators or semiconductors for electron in the other spin orientation. They have been the subject of extensive investigation, since the concept of half-metallicity was introduced by de Groot et al. [1]. Soon after their discovery, half-metals started being applied in technology, for example, as multilayer spin valves systems [2], spin injectors [3], or spin transistors [4], just to name few. Originally, halfmetallicity was reported in half-Heusler compounds, such as NiMnSb [1]; but in time, materials of different structures were found to have the same property. For example, halfmetallicity was predicted in many zinc-blende (ZB) compounds of transition elements with non-metals [4] as well as in rock-salt (RS) materials, such as $\mathrm{MnN}$ and $\mathrm{CrN}$ [5]. Half-metallic compounds of transition metals with group 15 elements, i.e., transition metal pnictides, especially attract much attention. First, their structure is commensurate with the structure of many semiconductors, which makes a process of multilayer fabrication of devices for spintronics easier and better controlled. Second, transition metal pnictides are characterized by a high Curie temper-

CThe Korean Magnetics Society. All rights reserved.

*Corresponding author: Tel: +82-32-860-7654

Fax: +82-32-872-7562, e-mail: jilee@inha.ac.kr ature, which allows maintaining the spin polarization at a high temperature and consequently stabilizing the properties of working devices.

In the search of half-metals, compounds of metals and chalcogens were investigated as well, and materials of the form TX, where T is a transition metal (e.g., V, Cr, Mn) and $\mathrm{X}$ is a group 16 element $(\mathrm{S}, \mathrm{Se}, \mathrm{Te})$, were reported to have promising half-metallic properties [4].

Group 14 elements might be as good components to use in designing new materials for spintronics as the elements of groups 15 and 16 . However, there has been much less reports on the electronic and the magnetic properties of carbides. Compounds such as $\mathrm{CaC}, \mathrm{SrC}, \mathrm{BaC}$ [6-9], $\mathrm{CaSi}$, and $\mathrm{CaGe}[10]$ have been predicted to be good candidates for application to spintronics. Some transition metal carbides, e.g., $\mathrm{VC}, \mathrm{CrC}$, and $\mathrm{MnC}$ [11], have also been found to be half-metals. Recently, attention has turn to ZB compounds with rare earth d-metals, such as Sc and Y [12-14]. YC was found to be a robust half-metal, with a small, 1.00 $\mu_{\mathrm{B}}$, magnetic moment per primitive cell [14]. Materials with a small magnetic moment are desirable, because they are source of weaker demagnetizing fields and are considered to be energetically more efficient.

Since the bulk properties of $\mathrm{YC}$ are very promising, we found it worthy to investigate whether the half-metallicity of the compound is preserved at its surfaces. Finding the answer is essential for future applications, as majority of nowadays electronic devices rely on surface properties of 
materials rather than on their bulk properties.

In this paper, we will discuss about the electronic and half-metallic properties of (001) and (110) surfaces of YC calculated with the use of a first-principles method.

\section{Computational Details}

Zinc-blende YC lattice constant has been reported as $5.65 \AA[10]$, and we adapted this value to model (001) and (110) surfaces of the compound. The surfaces are shown in Fig. 1.

We chose 9-layers slabs to model the surfaces. The four atomic layers on both sides of the center layer guarantee enough screening for the center of the slab to be treated as the YC bulk. The distances between the atomic layers were $1.413 \AA$ and $1.998 \AA$ for (001) and (110) surfaces, respectively. Since YC retains half-metallic properties even when the lattice constant is compressed by $3 \%$, small relaxation and reconstruction cannot affect much on the electronic properties of the system. Therefore, we calculated unrelaxed and unreconstructed surfaces only.

In order to resolve the electronic structure of the $\mathrm{YC}$ bulk and surface, the Kohn-Sham equation [15] was solved self-consistently in terms of the full-potential linearized augmented plane wave (FLAPW) method [16, 17], within generalized gradient approximation (GGA) of PerdewBurke-Ernzerhof type [18] to the exchange-correlation potential.

Lattice harmonics with $l \leq 8$ were employed to expand the charge density, the potential, and the wave functions inside the muffin-tin (MT) radii of 2.50 a.u. for $\mathrm{Y}$ and 1.50 a.u. for $\mathrm{C}$ atoms, respectively. The number of basis functions was about 200 per atom. Integration was performed over a $11 \times 11 \times 11$ mesh of k-points inside the irreducible three-dimensional Brillouin zone (BZ) for the bulk YC and over $7 \times 7$ mesh inside the two-dimensional BZ for the two-dimensional systems. All core electrons were treated fully relativistically, while valence states were treated scalar relativistically, without spin-orbit coupling.

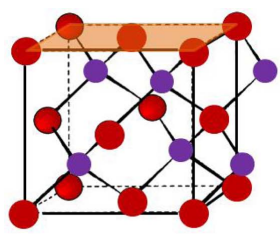

(a)

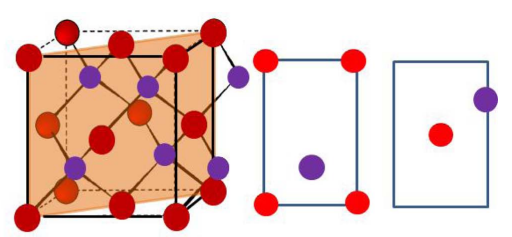

(b) (c)
Fig. 1. (Color online) A schematic view of a conventional unit cell of zinc-blende structured material with (a) (001) surface and (b) (110) surface highlighted; (c) relative positions of atoms in the top (left) and subsurface (right) layers of (110) surface. Different colors mark two types of atoms, i.e., Y and C.
Self-consistency was assumed when the difference between input and output charge densities was less than $1 \times 10^{-4}$ electrons/(a.u. $)^{3}$.

\section{Results and Discussion}

We preceded the calculation of the surface properties of ZB YC while checking whether our computational method gives results similar to those reported by $\mathrm{Wu}$ et al. [14]. We confirmed that the material is a half-metal, with the total magnetic moment (MM) of $1.00 \mu_{\mathrm{B}}$. We found that the energy gap is formed in the majority electrons channel, and it is $1.80 \mathrm{eV}$ wide. These findings perfectly agree with the reported values obtained within GGA. Also, similarly to the other findings, we found that the halfmetallic gap $\left(\mathrm{E}_{\mathrm{HM}}\right)$ is very narrow. The calculated magnetic moments (MMs) on $\mathrm{Y}$ and $\mathrm{C}$ sites are $0.12 \mu_{\mathrm{B}}$ and $0.41 \mu_{\mathrm{B}}$, respectively. In further analysis, when we compare the surface properties with the bulk properties, we shall refer to the results of our calculations of the bulk YC properties.

In Fig. 2, we present plots of atom-projected density of electronic states (DOS) calculated for (001) surface of the
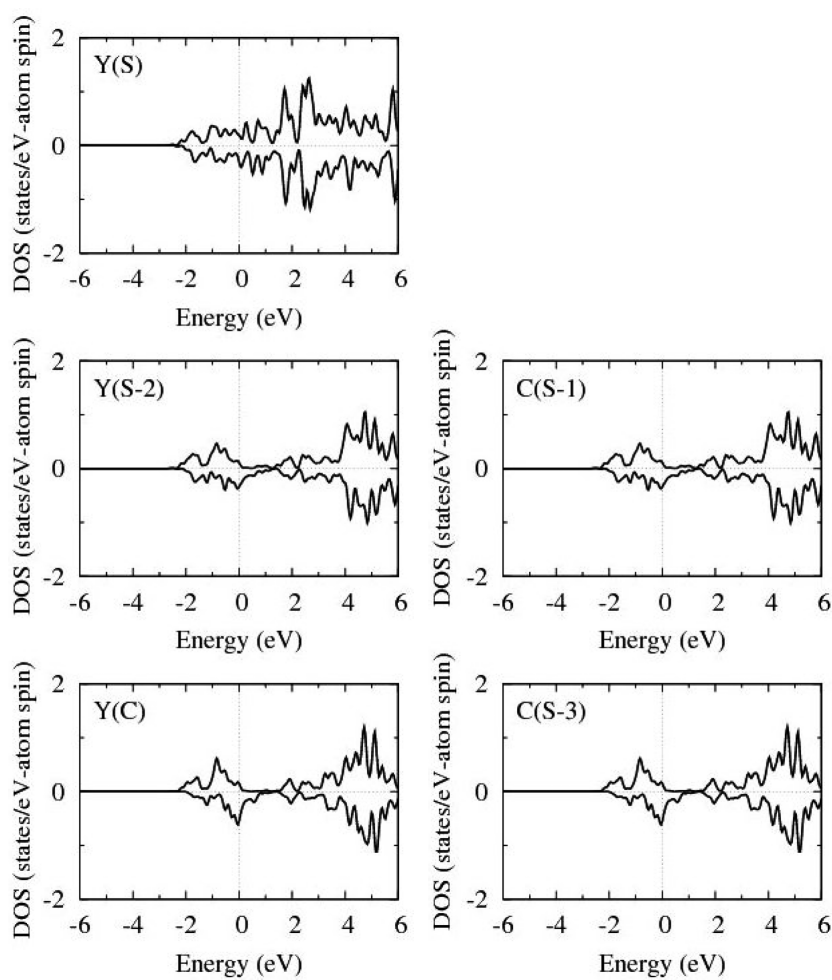

Fig. 2. Spin polarized density of states for $\mathrm{Y}$ and $\mathrm{C}$ atoms in the center $(\mathrm{C})$, the surface $(\mathrm{S})$, and subsurface layers denoted as (S-1), (S-2), and (S-3) of Y-terminated (001) YC surface. The spin-down states are factored by -1 and the Fermi levels are set to zero. 
YC. (001) surface is a polar surface, and it has two possible terminations: one with $\mathrm{Y}$ atoms and the other with $\mathrm{C}$ atoms in the topmost layer. The plots shown in Fig. 2 were calculated for Y-terminated surface. The expressions in parentheses refer to respective monolayers of the surface; $\mathrm{S}$ denotes the topmost layer, and S-n ( $\mathrm{n}=$ $1,2,3)$ marks deeper layers starting from subsurface $(\mathrm{n}=$ 1) and moving toward the center layer, C. It is evident that the half-metallicity of the bulk structure is destroyed at this surface; electron states are present at the Fermi level $\left(E_{F}\right)$ in both spin-up and spin-down channels. The loss of half-metallicity is confirmed by the calculated non-integer total magnetic moment of the system; and its value is $2.59 \mu_{\mathrm{B}}$. The density of states distribution in energy scale evolves from the center to the surface layer. Not surprisingly, the local DOS projected on $\mathrm{Y}$ atom in the center layer resembles that calculated for $\mathrm{Y}$ atom in the bulk structure. As far as the Y-projected DOS calculated for the topmost layer are concerned, the DOS is much different from that calculated for $\mathrm{Y}$ in the bulk; the occupied states are more delocalized and the larger number of unoccupied states arises due to the presence of unsaturated bonds. There is also a striking symmetry in the DOS plots calculated for spin-up and spin-down electrons, which suggests that the magnetic properties of $\mathrm{Y}$ atoms at the surface are greatly suppressed. Comparison of the DOSs plotted for $\mathrm{C}(\mathrm{S}-3)$ and $\mathrm{C}(\mathrm{S}-1)$ atoms leads to a conclusion that the electronic structure of $\mathrm{C}$ atoms is not as much affected by the surface termination; the calculated DOSs projected on $\mathrm{C}(\mathrm{S}-1)$ atoms have similar features to the DOSs calculated for $\mathrm{C}(\mathrm{S}-3)$ atom, which we can consider a bulk-like atom. The C(S-1) and Y(S-2) DOS maxima are at the same energy, which means that the nature of bonding between $\mathrm{Y}$ and $\mathrm{C}$ atoms of subsequent layers is the same as in the bulk structure. The surface termination does not change the process of mixing between the $\mathrm{p}$ orbitals of $\mathrm{C}$ and $\mathrm{d}$ orbitals of $\mathrm{Y}$, although the interactions are not as strong as in the bulk. Quantitatively, the number of both occupied and unoccupied C(S1) states is smaller than in bulk-like $\mathrm{C}(\mathrm{S}-3)$ layer, and the spin-up and spin-down occupied states are more evenly distributed. This effect is due to the delocalization of Y(S) electrons at the surface, which causes a slight redistribution of electron charge in $\mathrm{C}(\mathrm{S}-1)$ layer. The charge distribution between the layers of the surface will also be analyzed in this section.

In Fig. 3, we present similar results to those shown in Fig. 2, but they were calculated for C-terminated (001) YC surface. At this surface, half-metallicity is also destroyed. The Fermi level no longer lies within the energy gap, and the calculated total MM is $7.76 \mu_{\mathrm{B}}$. The changes
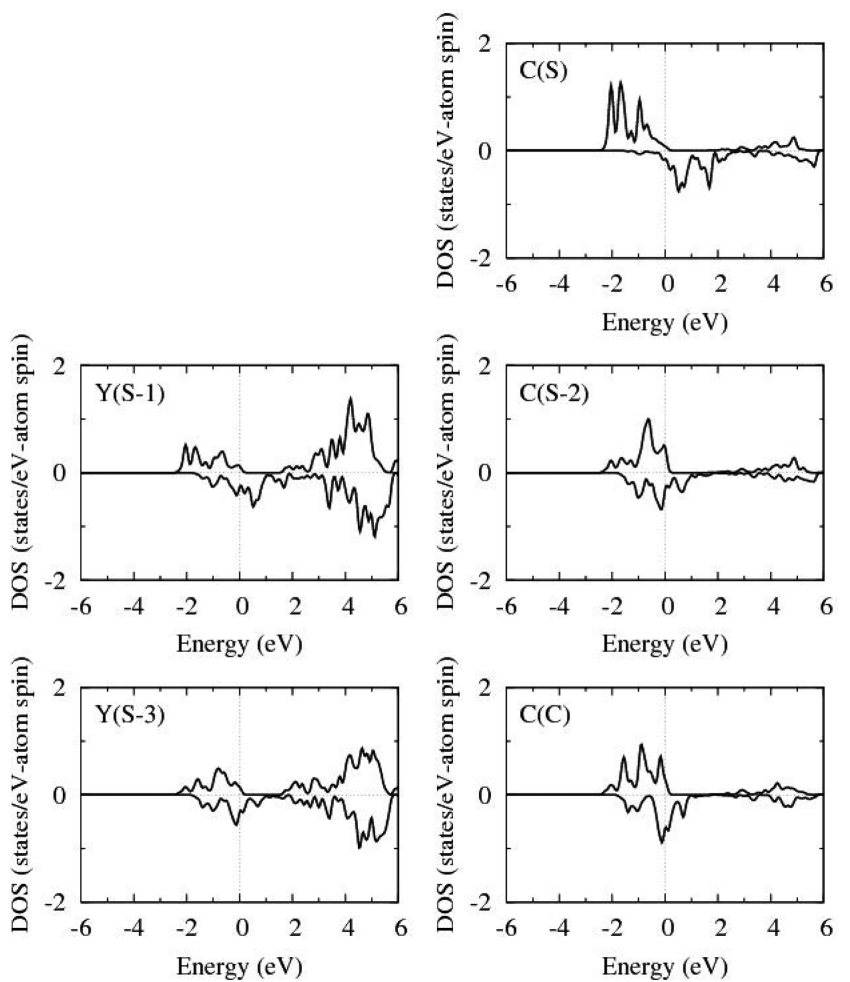

Fig. 3. Spin polarized density of states for $\mathrm{Y}$ and $\mathrm{C}$ atoms in the center $(\mathrm{C})$, the surface $(\mathrm{S})$, and subsurface layers denoted as (S-1), (S-2), and (S-3) of C-terminated (001) YC surface. The spin-down states are factored by -1 and the Fermi levels are set to zero.

in distribution of DOS attributed to $\mathrm{Y}$ atoms in various layers are not as dramatic as in the case of Y-terminated surface, although there is a noticeable increase in the number of unoccupied states associated with Y(S-1) atom. However, since this atom does not have unsaturated bonds, screening from $\mathrm{C}(\mathrm{S})$ atom is enough to prevent considerable changes in DOS distribution. Certainly, there should be an impact on the DOSs contributed by $\mathrm{C}$ atoms. Interestingly, the changes in DOS contributed by $\mathrm{C}$ atoms of the C-terminated surface must have different origin than the changes observed in the DOSs of $\mathrm{Y}$ atoms in Yterminated surface. The most striking difference is that in the Y-terminated surface the number of states associated with the atom at the topmost layer was built up without a large shift in energy, as compared with the atoms in the deeper layers. This is different in the case of C-terminated surface; we found that at the surface, no spin-down states of $\mathrm{C}(\mathrm{S})$ atoms are occupied. This must result in an increase of the magnetic moment of $\mathrm{C}(\mathrm{S})$ atom in this system.

In Table 1, we present the 1-decomposed majority and minority spin electrons inside each MT sphere, as well as the calculated magnetic moments for each atom in Yterminated and C-terminated YC (001) surfaces. For com- 
Table 1. 1-decomposed majority and minority spin electrons inside each muffin-tin sphere, and the magnetic moments (MM) on each atom calculated for bulk YC and for (001) YC surfaces.

\begin{tabular}{|c|c|c|c|c|c|}
\hline Atom & $\stackrel{\mathrm{s}}{\uparrow / \downarrow}$ & $\underset{\uparrow / \downarrow}{\mathrm{p}}$ & $\underset{\uparrow / \downarrow}{\mathrm{d}}$ & $\begin{array}{l}\text { Total } \\
\uparrow / \downarrow\end{array}$ & $\begin{array}{l}\mathrm{MM} \\
{\left[\mu_{\mathrm{B}}\right]}\end{array}$ \\
\hline \multicolumn{6}{|c|}{ Bulk YC } \\
\hline $\mathrm{Y}$ & $0.067 / 0.065$ & $0.100 / 0.067$ & $0.384 / 0.311$ & $0.570 / 0.455$ & 0.116 \\
\hline $\mathrm{C}$ & $0.468 / 0.455$ & $0.844 / 0.444$ & $0.000 / 0.000$ & $1.312 / 0.899$ & 0.413 \\
\hline \multicolumn{6}{|c|}{ Y-terminated (001) YC } \\
\hline $\mathrm{Y}(\mathrm{S})$ & $0.072 / 0.070$ & $0.050 / 0.044$ & $0.362 / 0.315$ & $0.493 / 0.435$ & 0.057 \\
\hline $\mathrm{Y}(\mathrm{S}-2)$ & $0.064 / 0.062$ & $0.094 / 0.074$ & $0.368 / 0.328$ & $0.544 / 0.478$ & 0.068 \\
\hline $\mathrm{Y}(\mathrm{C})$ & $0.066 / 0.066$ & $0.097 / 0.067$ & $0.375 / 0.331$ & $0.557 / 0.476$ & 0.081 \\
\hline $\mathrm{C}(\mathrm{S}-1)$ & $0.454 / 0.450$ & $0.723 / 0.612$ & $0.000 / 0.000$ & $1.183 / 1.062$ & 0.121 \\
\hline $\mathrm{C}(\mathrm{S}-3)$ & $0.463 / 0.450$ & $0.848 / 0.488$ & $0.000 / 0.000$ & $1.311 / 0.939$ & 0.372 \\
\hline \multicolumn{6}{|c|}{ C-terminated (001) YC } \\
\hline $\mathrm{Y}(\mathrm{S}-1)$ & $0.070 / 0.062$ & $0.099 / 0.054$ & $0.431 / 0.252$ & $0.622 / 0.377$ & 0.245 \\
\hline $\mathrm{Y}(\mathrm{S}-3)$ & $0.066 / 0.064$ & $0.097 / 0.069$ & $0.382 / 0.313$ & $0.565 / 0.458$ & 0.107 \\
\hline $\mathrm{C}(\mathrm{S})$ & $0.504 / 0.461$ & $1.112 / 0.071$ & $0.001 / 0.000$ & $1.616 / 0.532$ & 1.084 \\
\hline $\mathrm{C}(\mathrm{S}-2)$ & $0.459 / 0.448$ & $0.825 / 0.518$ & $0.000 / 0.000$ & $1.284 / 0.966$ & 0.318 \\
\hline $\mathrm{C}(\mathrm{C})$ & $0.464 / 0.451$ & $0.869 / 0.460$ & $0.000 / 0.000$ & $1.334 / 0.911$ & 0.423 \\
\hline
\end{tabular}

parison, we also included the decomposed charges on atoms in the bulk YC.

The atoms of the center and S-3 layers are well screened from the surface, so the calculated charges on $\mathrm{Y}(\mathrm{C})$, $\mathrm{C}(\mathrm{S}-3)$ of Y-terminated surface, as well as those on $\mathrm{C}(\mathrm{C})$ and $\mathrm{Y}(\mathrm{S}-3)$ of C-terminated surface, do not differ much from the charges on $\mathrm{Y}$ and $\mathrm{C}$ atoms of the bulk structure. It is worth mentioning here that the magnetic properties of the bulk YC are largely contributed by the electrons of $\mathrm{C}$ atom.

In the Y-terminated (001) surface the total number of electrons on $\mathrm{Y}$ atom at the surface decreases, regardless of their spin. The total number of spin-up electrons of $\mathrm{C}$ atom near the surface increases, while the total number of spin-down electrons of the atom decreases. Due to the surface termination, the calculated $\mathrm{MM}$ on $\mathrm{Y}(\mathrm{S})$ atom decreases to $0.057 \mu_{\mathrm{B}}$ from the bulk value of $0.081 \mu_{\mathrm{B}}$, as calculated for $\mathrm{Y}(\mathrm{C})$ atom. This decrease is due to a delocalization of the electrons at the surface. Since the Y atoms of the topmost layer have unsaturated bonds, the valence d-electrons are spilled out to the vacuum region forming space charge layer that screens the surface termination. Compared with the atoms of the center layer, $\mathrm{Y}(\mathrm{S})$ atom loses 0.064 spin-up electrons and 0.041 spindown electrons. The change is caused by the redistribution of p-electrons rather than d-electrons. The number of spin-up p-electrons on $\mathrm{Y}(\mathrm{S})$ atom is 0.050 while on $\mathrm{Y}(\mathrm{C})$ atom it is 0.097 . Also, the number of spin-down electrons decreases from 0.067 to 0.044 . The changes in the numbers of d-electrons are in the same direction, although they are smaller in magnitude. Apart from the electrons spilled out to the vacuum region, some charges are back-donated to the previously empty states of $\mathrm{C}$ atoms. As a result, the occupation of $\mathrm{C}(\mathrm{S}-1)$ spin-down states increases, as compared with the bulk $\mathrm{C}$ atoms, and the calculated MM on $\mathrm{C}(\mathrm{S}-1)$ atom decreases to $0.121 \mu_{\mathrm{B}}$. The overall result is that the ferromagnetism of Y-term YC (001) surface is much weaker than of the bulk material.

The obvious conclusion from a comparison of the calculated $\mathrm{MM}$ on atoms of C-terminated (001) YC surface, also presented in Table 1, is that the surface termination defines the magnetic properties of the system. In the C-terminated surface, $\mathrm{C}(\mathrm{S})$ atom has a $\mathrm{MM}$ of $1.084 \mu_{\mathrm{B}}$, which is more than twice larger than the MM on $\mathrm{C}$ atom in the center layer. Also, the total MM on Y(S1) atom is increased to $0.245 \mu_{\mathrm{B}}$ from $0.107 \mu_{\mathrm{B}}$, as calculated for $\mathrm{Y}(\mathrm{S}-3)$. The broken bonds on $\mathrm{C}$-terminated surface are of different nature than those on Y-terminated surface. The electrons of $\mathrm{C}$ atoms are strongly bounded to the nuclei, and they do not easily cross the energy barrier to be spread to vacuum. Instead, the electrons taking part in a bond formation with $\mathrm{Y}$ atoms in deeper layers tend to occupy available p-states of the parent atoms. Since it is energetically more favorable to occupy spin-up states, the charges are redistributed from spin-down to spin-up electron levels. As a result, the magnetic properties of the surface are enhanced.

It is not only structurally but also qualitatively different 

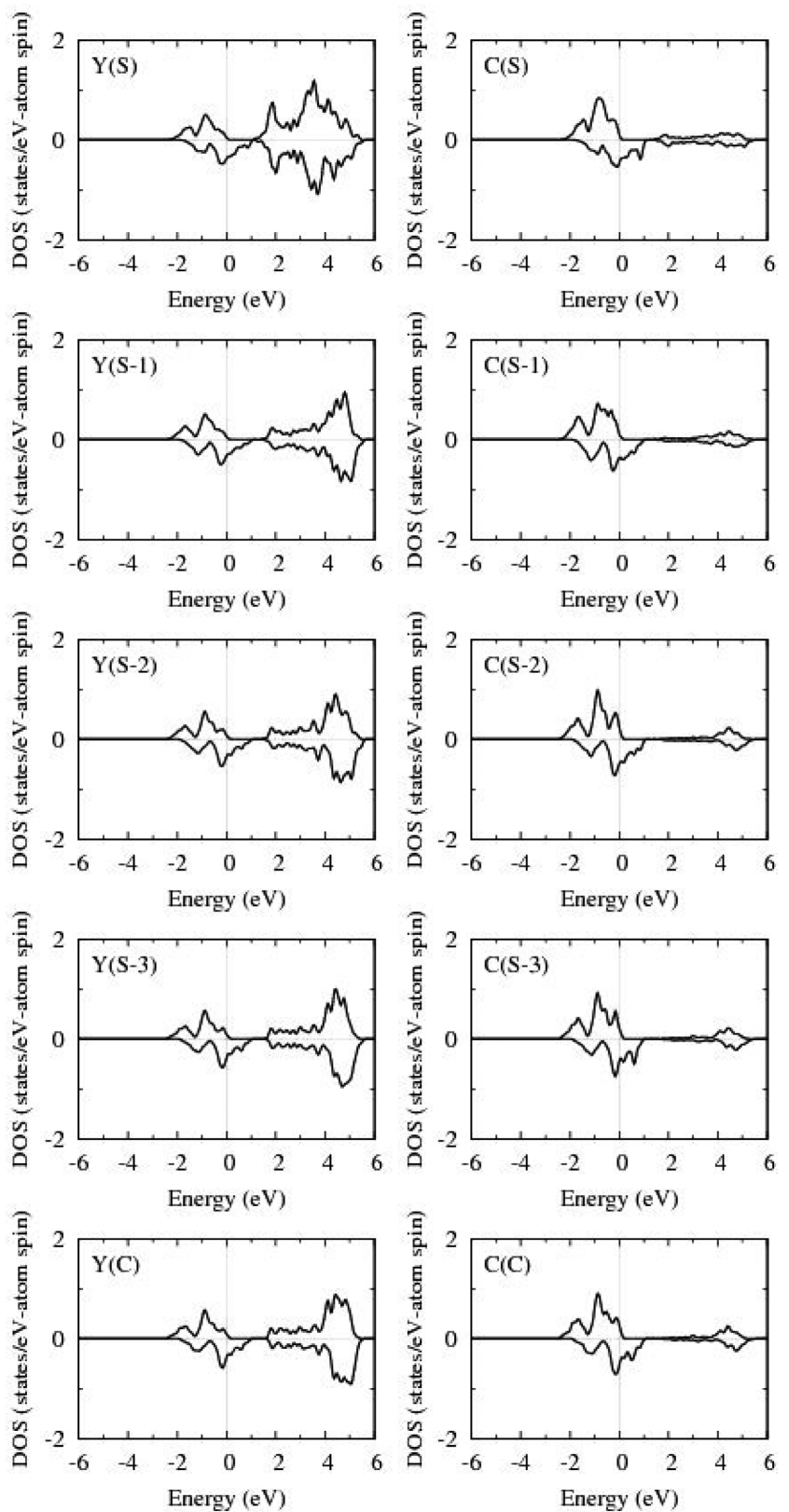

Fig. 4. Spin polarized density of states for $\mathrm{Y}$ and $\mathrm{C}$ atoms in the center $(\mathrm{C})$, the surface $(\mathrm{S})$, and subsurface layers denoted as (S-1), (S-2), and (S-3) of (110) YC surface. The spin-down states are factored by -1 and the Fermi levels are set to zero.

between (110) surface of ZB materials and (001) surface. This is because (110) is a nonpolar surface terminated by a layer composed of both types of atoms. However, the nonpolarity of the surface does not sustain half-metallicity of YC. In Fig. 4, we present plots of atom-projected DOSs calculated for the (110) YC surface; it is clear that similar to the case of (001) surfaces, the half-metallicity of the bulk is destroyed. The number of states present at the Fermi level is not large, but it is enough to make the surface metallic. The total MM calculated for the surface is $8.81 \mu_{\mathrm{B}}$. Interestingly, the density of occupied states contributed by $\mathrm{Y}$ atom in subsequent layers does not change much as we move from the center of the slab toward the vacuum. The most distinct feature of the total DOS of $\mathrm{Y}(\mathrm{S})$ atom is considerably larger number of unoccupied states, as compared with the $\mathrm{Y}$ atoms of other layers. In the (110) YC surface, the states contributed by the electrons of $Y$ atoms are not much more delocalized at the topmost layer than in the bulk; and the same may be stated about the states contributed by $\mathrm{C}$ atoms. It implies that surface termination does not affect the magnetic properties of $\mathrm{YC}$, and at this surface they remain very similar to the properties of bulk structure. It also implies that in-plane hybridization of $\mathrm{C}$ and $\mathrm{Y}$ orbitals is stronger than the mixing of out-of-plane orbitals in the direction normal to the surface.

In Table 2, the 1-decomposed spin-up and spin-down electrons inside MT sphere of the atoms in (110) YC surface and the values of MMs on each atom are collected. The values of the charges inside of each MT are relatively small, just as they were in (001) surfaces; so, we can conclude that a large amount of charge is distributed into the interstitial region, the volume of which is relatively large in non-closely packed structures. The amount of charge localized on $\mathrm{Y}(\mathrm{S})$ atom of (110) surface is between the values calculated for the $\mathrm{Y}(\mathrm{S})$ atom of $\mathrm{Y}$-terminated (001) surface and for the Y(S-1) atom of the C-terminated (001) surface. This is in agreement with expectations based on the structural properties of (110) surface; the number of dangling bonds at (110) surface is smaller than at (001) surface, so less electrons become delocalized. The same may be stated about the total charges localized on $\mathrm{C}(\mathrm{S})$ atoms, which supports the conclusion that inplane interaction between the electrons of $\mathrm{Y}$ and $\mathrm{C}$ atoms are decisive for the properties of the surface. Interestingly, the MMs calculated for the atoms of the topmost layer do not differ considerably from those on atoms of layer other than the subsurface layer. While the MM on $\mathrm{Y}(\mathrm{S})$ atom is $0.102 \mu_{\mathrm{B}}$, which is exactly the same as the MM on the $\mathrm{Y}(\mathrm{C})$ atom and very close to the MMs of $\mathrm{Y}$ atoms in (S2) and (S-3) layers, the MM of the $\mathrm{Y}(\mathrm{S}-1)$ atom is 0.094 $\mu_{\mathrm{B}}$. The effect is even stronger as $\mathrm{C}$ atoms are concerned. The $\mathrm{MM}$ on $\mathrm{C}(\mathrm{S})$ atom is $0.457 \mu_{\mathrm{B}}$ and that on $\mathrm{C}(\mathrm{S}-1)$ atom is $0.346 \mu_{\mathrm{B}}$. This indicates that the range of surface effects is greater in (110) surface than in (001) surfaces. Another difference is opposite effect of the surface on the magnetic properties of the system; the magnetism of (110) surface is enhanced as compared with the bulk properties. The same effects were found in other studies of polar and non-polar surfaces of ZB and rock salt 
Table 2. 1-decomposed majority and minority spin electrons inside of each muffin-tin sphere, and the magnetic moments (MM) on each atom as calculated for (110) YC surface.

\begin{tabular}{|c|c|c|c|c|c|}
\hline Atom & $\begin{array}{c}\mathrm{S} \\
\uparrow / \downarrow\end{array}$ & $\begin{array}{c}\mathrm{p} \\
\uparrow / \downarrow\end{array}$ & $\begin{array}{c}\mathrm{d} \\
\uparrow / \downarrow\end{array}$ & $\begin{array}{c}\text { Total } \\
\uparrow / \downarrow\end{array}$ & $\begin{array}{l}\mathrm{MM} \\
{\left[\mu_{\mathrm{B}}\right]}\end{array}$ \\
\hline $\mathrm{Y}(\mathrm{S})$ & $0.065 / 0.064$ & $0.070 / 0.051$ & $0.389 / 0.313$ & $0.538 / 0.436$ & 0.102 \\
\hline $\mathrm{Y}(\mathrm{S}-1)$ & $0.066 / 0.064$ & $0.097 / 0.070$ & $0.377 / 0.320$ & $0.559 / 0.465$ & 0.094 \\
\hline $\mathrm{Y}(\mathrm{S}-2)$ & $0.066 / 0.064$ & $0.097 / 0.067$ & $0.381 / 0.317$ & $0.564 / 0.459$ & 0.105 \\
\hline $\mathrm{Y}(\mathrm{S}-3)$ & $0.067 / 0.065$ & $0.097 / 0.066$ & $0.383 / 0.316$ & $0.567 / 0.458$ & 0.109 \\
\hline $\mathrm{C}(\mathrm{S})$ & $0.472 / 0.454$ & $0.844 / 0.404$ & $0.001 / 0.001$ & $1.316 / 0.859$ & 0.457 \\
\hline $\mathrm{C}(\mathrm{S}-1)$ & $0.462 / 0.451$ & $0.823 / 0.490$ & $0.000 / 0.000$ & $1.286 / 0.941$ & 0.346 \\
\hline $\mathrm{C}(\mathrm{S}-2)$ & $0.466 / 0.452$ & $0.852 / 0.456$ & $0.000 / 0.000$ & $1.318 / 0.909$ & 0.410 \\
\hline $\mathrm{C}(\mathrm{S}-3)$ & $0.466 / 0.452$ & $0.851 / 0.457$ & $0.000 / 0.000$ & $1.317 / 0.910$ & 0.408 \\
\hline $\mathrm{C}(\mathrm{C})$ & $0.466 / 0.452$ & $0.850 / 0.458$ & $0.000 / 0.000$ & $1.316 / 0.911$ & 0.405 \\
\hline
\end{tabular}

structured MgN [19], except that the half-metallicity was retained in the pnictide.

\section{Conclusions}

We studied the surface effects on the electronic and magnetic properties of YC in zinc-blende structure, which, as a bulk material, is a half-metal The objects of the study were both polar (001) surfaces and nonpolar (110) surface. We found that half-metallicity of the bulk is destroyed at each studied surface. Additionally, the magnetic properties of Y-terminated (001) surface are suppressed. While the bulk the magnetic moment on $\mathrm{Y}$ atom is $0.116 \mu_{\mathrm{B}}$, the value calculated for $\mathrm{Y}(\mathrm{S})$ atom in Y-terminated (001) surface is only $0.057 \mu_{\mathrm{B}}$. In the case of (110) YC surface, the magnetic properties seem to be slightly enhanced at the topmost layer, due to an increased occupation of spinup $\mathrm{C} \mathrm{p}$ orbitals. This is due to redistribution of electronic charge between $p$ orbitals of $\mathrm{C}(\mathrm{S}-1)$ atoms, i.e., in the outof-plane direction, as well as between in-plane $\mathrm{p}$ orbitals of $\mathrm{Y}(\mathrm{S})$ and $\mathrm{C}(\mathrm{S})$ atoms. The $\mathrm{C}$-terminated (001) surface exhibits the strongest magnetization of $\mathrm{C}$ atoms of the topmost layer. The calculated magnetic moment is 1.084 $\mu_{\mathrm{B}}$, while the magnetic moment of the $\mathrm{C}$ atoms in the bulk is only $0.423 \mu_{\mathrm{B}}$.

We found that half-metallicity of bulk YC is destroyed at the surfaces. In Y-terminated (001) surface, the electrons of $\mathrm{Y}$ atom of the topmost layer are strongly delocalized that there is practically no energy gap in any energy range. In the other two surfaces, we found rather a small number of states at the Fermi level which are followed by a range of energy with no presence of unoccupied states. However, in our opinion, the number of occupied states at the Fermi level may be increased in materials working in real conditions under non-zero temperature. Therefore, from the point of view of applying this material to spintronics, our findings may be discouraging.

\section{Acknowledgement}

This work was supported by the Inha University Research Fund.

\section{References}

[1] R. A. de Groot, F. M. Mueller, P. G. van Engen, and K. H. J. Bushow, Phys. Rev. Lett. 50, 2024 (1983).

[2] F. Yang, Z. Kang, X. Chen, and Y. Xue, J. Phys. D: Appl. Phys. 46, 325003 (2013).

[3] S. Chadov, T. Graf, K. Chadova, X. Dai, G. H. Fecher, and C. Felser, Phys. Rev. Lett. 107, 047202 (2011).

[4] I. Galanakis and P. Mavropoulos, Phys. Rev. B 67, 104417 (2003).

[5] M. S. Miao and W. R. L. Lambrecht, Phys. Rev. B 71, 214405 (2005).

[6] G. Y. Gao, K. L. Yao, E. Şaşıŏlu, L. M. Sandratskii, Z. L. Liu, and J. L. Jiang, Phys. Rev. B 75, 174442 (2007).

[7] G. Y. Gao and K. L. Yao, J. Appl. Phys. 106, 053703 (2009).

[8] G. Y. Gao and K. L. Yao, Appl. Phys. Lett. 91, 082512 (2007).

[9] Q. S. Shao and H. Zhao, J. Supercond. Nov. Magn. 25, 2063 (2012).

[10] G. Y. Gao, K. L. Yao, Z. L. Liu, J. L. Jiang, L. H. Yu, and Y. L. Shi, J. Phys.: Condens. Matt. 19, 315222 (2007).

[11] J. E. Pask, L. H. Yang, C. Y. Fong, W. E. Pickett, and S. Dag, Phys. Rev. B 67, 224420 (2003).

[12] Y. Xing, Y. Liu, S. N. Li, Y. H. Zhao, and W. H. Xie, Phys. Stat. Sol. B 247, 2268 (2010).

[13] S. W. Fan, L. J. Ding, Z. L. Wang, and K. L. Yao, Appl. Phys. Lett. 102, 022404 (2013).

[14] Q. Wu, Z. Wang, S. Fan, and K. Yao, J. Magn. Magn. 
Mater. 368, 44 (2014).

[15] W. Kohn and L. J. Sham, Phys. Rev. A 140, 1133 (1965).

[16] E. Wimmer, H. Krakauer, M. Weinert, and A. J. Freeman, Phys. Rev. B 24, 6864 (1981), and references therein.

[17] M. Weinert, E. Wimmer, and A. J. Freeman, Phys. Rev.
B 26, 4571 (1982).

[18] J. P. Perdew, K. Burke, and M. Ernzerhof, Phys. Rev. Lett. 77, 3865 (1996).

[19] B. Bialek and J. I. Lee, Solid State Commun. 150, 2138 (2010). 